\title{
CARTOGRAFÍA DE PRECIPITACIONES Y TEMPERATURAS EN EL VALLE MEDIO DEL EBRO MEDIANTE LA UTILIZACIÓN DE DIFERENTES TÉCNICAS ESTADÍSTICAS*
}

\author{
Sergio Martín Vicente Serrano y Miguel Ángel Saz Sánchez \\ Dpto. de Geografía y Ordenación del Territorio. Universidad de Zaragoza. \\ Campus de San Francisco, 50009-Zaragoza. e-mail: svicen@posta.unizar.es
}

\begin{abstract}
Resumen: Este trabajo analiza la calidad final de diferentes cartografías continuas de precipitaciones y temperaturas realizadas a partir de distintos métodos de interpolación. El análisis se ha realizado en el sector central del valle del Ebro, un espacio de topografía suave pero que se encuentra dominado por complejos patrones climáticos. Se han realizado 23 cartografías con diferentes tipos de métodos (locales, globales y geoestadísticos). Los mejores resultados en la cartografía de precipitaciones se han obtenido mediante la aplicación de métodos geoestadísticos, en concreto las técnicas de block-kriging y cokriging. Las temperaturas se han cartografiado con mejores resultados a partir de un modelo de regresión múltiple. Se discute acerca de la utilidad de las diferentes técnicas cartográficas en función de la variable analizada.
\end{abstract}

Palabras clave: Cartografía, métodos de interpolación, métodos geoestadísticos, temperaturas, precipitaciones, Valle del Ebro, España.

\begin{abstract}
This work analyses the final quality of different continuous cartographies of precipitation and temperatures carried out using different interpolation techniques. The analysis has been performed in the central Ebro valley, an area with flat topography but complex climatic patterns. 23 maps of temperature and precipitation have been performed with different methods (local, global and geostatistics). The best results in the precipitation maps have been obtained by means of geostatistical methods. Block-kriging and cokriging provide the best results for the interpolation of precipitation. Regression model provicte the higher quality results for temperature interpolation. The utility of different mapping methods is discussed.
\end{abstract}

Key words: Cartography, Interpolation methods, geostatistics, temperatures, precipitation, Ebro valley, Spain.

* Recibido: 25-3-2002. Aceptado: 11-4-2002 


\section{Introducción}

La demanda de cartografías climáticas por parte de múltiples disciplinas encuentra una serie de problemas relacionados con la cobertura espacial de los datos. La deficiente cobertura espacial de la información climática, relacionada con el carácter puntual de los datos y la escasa densidad de observatorios existentes, es una limitación importante, en ocasiones determinante, para la puesta en marcha de investigaciones o la toma de decisiones ligadas a la ordenación agrícola y ambiental. El problema se agudiza en los países del tercer mundo o en los grandes vácíos poblacionales de la tierra (desiertos, selvas, regiones polares), donde la red de observatorios es muy poco densa. En los países mediterráneos, debido a la dispersión en el hábitat rural, también se suelen encontrar problemas en la obtención de datos climatológicos que permitan realizar adecuadas cartografías.

Para solucionar este problema se han desarrollado diferentes métodos estadísticos que permiten obtener una estimación continua en el territorio, para posibilitar la realización de mapas climáticos mediante la estimación del valor de las variables climáticas en los puntos en los que no se dispone de información. Existen metodologías muy diversas en cuanto a su concepción y su formulación matemática (Borrough y McDonnell, 1998). Estos métodos han sido ampliamente aplicados a la interpolación espacial de datos climáticos para la obtención de cartografías (Ninyerola et al., 2000; Goovaerts, 1997; Salas y Chuvieco, 1993).

En la bibliografía encontramos mayor interés por la realización de cartografías en sectores montañosos (Goovaerts, 1999; Prudhomme y Reed, 1998; Daly et al., 1994). Este hecho está motivado por los problemas que provoca la complejidad del relieve, ya que genera múltiples microambientes climáticos cuyos valores son difíciles de cartografiar. A esto se une la escasa red de observatorios que encontramos en estas áreas, ya que están escasamente pobladas y los observatorios meteorológicos se localizan en los fondos de valle.

Las dificultades son menores en las regiones llanas. No obstante, hay que tener en cuenta que muchas de las depresiones del mundo tienen unas características climáticas muy complejas, debido a su posición respecto a los relieves circundantes, a las variaciones estacionales de los flujos dominantes y al frecuente embolsamiento de aire frio en el centro de los valles, con fenómenos de inversiones térmicas y abundantes precipitaciones horizontales. Esta complejidad provoca variaciones climáticas muy contrastadas entre espacios cercanos, lo que dificulta la cartografía de las variables climáticas en los puntos en los que no se dispone de información. Es indudable que la cartografía en estas áreas presenta menores problemas que en las regiones montañosas. No obstante, una correcta realización de cartografías climáticas en áreas llanas tiene un enorme interés científico y aplicado, ya que la mayor parte de las acti- 
vidades agrarias se concentran en los llanos, al igual que la mayor parte de la población y de sus actividades económicas y lúdicas.

Por esta razón, en este trabajo se plantea la realización de distintas cartografías mediante diferentes metodologías en un espacio llano, pero climáticamente complejo, como es la depresión del Ebro (NE de España). Los métodos se aplican a dos variables climáticas: temperaturas y precipitaciones, con la finalidad de determinar las diferencias que se obtienen a partir de cada método y comprobar cual de ellos es más válido.

\section{2. Área de estudio}

En la figura 1 se indica la localización del área de estudio. Se trata de un área de $22.909 \mathrm{~km}^{2}$, limitada al norte por las sierras prepirenaicas, al sur por la cordillera ibérica, y al este y oeste por los límites administrativos de la comunidad autónoma de Aragón. El sector central del valle del Ebro (NE de España) constituye un buen ejemplo de espacio con topografía suave pero en el que el comportamiento de las variables climáticas ofrece un elevado grado de complejidad espacial. Se trata de un área donde domina la presencia de plataformas estructurales horizontales elaboradas sobre materiales terciarios, con altitudes inferiores a $500 \mathrm{~m}$. Terrazas y glacis cuaternarios limitan cuencas aluviales de fondo plano (Pellicer y Echeverría, 1990). En el sector septentrional se alcanzan altitudes superiores a los $1500 \mathrm{~m}$, al enlazar con la vertiente meridional de las Sierras Exteriores Pirenaicas. Al sur se encuentran las sierras del Sistema Ibérico, con más de $2.300 \mathrm{~m}$ en el macizo del Moncayo, y más de $1.500 \mathrm{~m}$ en los Puertos de Beceite, en el extremo más suroriental.

Latitudinalmente se encuentra dentro del dominio climático subtropical, en el contacto entre las influencias templadas y subtropicales. El relieve actúa como elemento que aísla el valle respecto a la influencia marítima, por lo que el clima presenta importantes matices de continentalidad. Todo ello determina la complejidad del clima en la zona de estudio, en la que nos aparece como principal rasgo definitorio la aridez (Ascaso y Casals, 1981; Cuadrat, 1989; Cuadrat, 1991; Pérez y Creus, 1994; Creus y Ferraz, 1995; Creus 1996; Del Valle, 1996).

Los totales anuales de precipitación oscilan entre 300 y $450 \mathrm{~mm}$, aunque pueden superarse los $800 \mathrm{~mm}$ en las áreas montañosas septentrionales y meridionales. Estos reducidos volúmenes van unidos a una elevada variabilidad interanual. Así, frente a años donde las precipitaciones superan ampliamente el valor promedio, se observan otros donde lo recogido no llega ni siquiera a un tercio de esa cantidad. La temperatura media anual oscila entre los $14^{\circ} \mathrm{C}$ y los $16^{\circ} \mathrm{C}$ en las zonas llanas y por debajo de los $11^{\circ} \mathrm{C}$ en los sectores más elevados. Las temperaturas promedio son suaves pero 
la oscilación térmica absoluta es muy acusada, ya que en los meses estivales no es extraño que se registren máximas que superen $\operatorname{los} 40^{\circ} \mathrm{C}$, y en invierno registros inferiores a $-10^{\circ} \mathrm{C}$, como consecuencia del elevado grado de continentalidad que propicia su situación geográfica (Cuadrat, 1999).

La vegetación dominante es esteparia de influencia térmica (Pedrocchi, 1998; Suárez et al., 1992). Esta vegetación está condicionada por la litología, los suelos y la aridez característica. Si excluimos las escasas áreas montañosas, en las que incluso aparecen pequeñas formaciones de Fagus syluatica, Pinus syluestris, Quercus petraea y Q. Pyrenaica, la vegetación arbórea natural se encuentra en proceso de regresión debido a la intensa actividad humana, quedando pequeños bosques de Juniperus thurifera L, Quercus ilex ssp ballota y $Q$. coccifera., localizados generalmente en los taludes de los relieves tabulares, junto a algunas repoblaciones de Pinus balepensis.

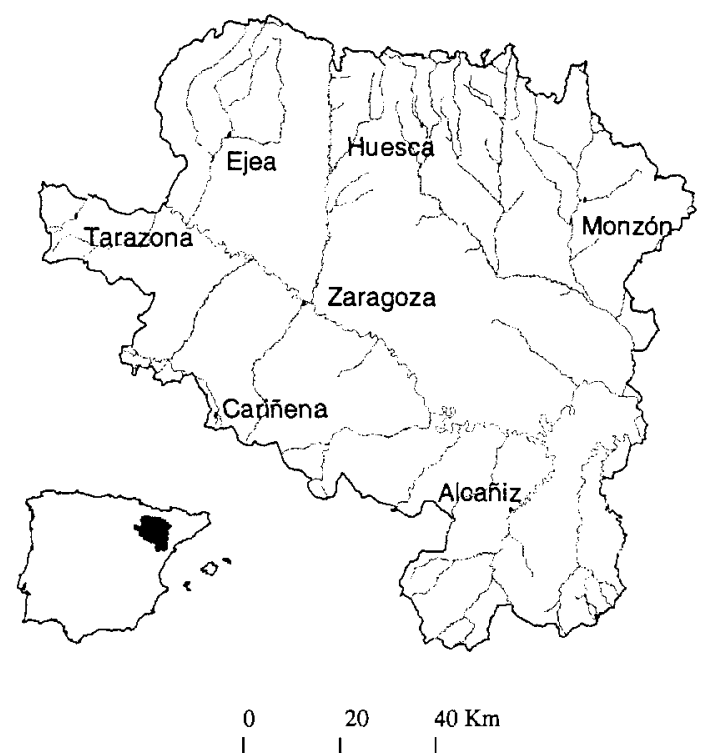

Figura 1. Localización del área de estudio.

\section{Metodología}

\subsection{Datos utilizados}

Para la realización de las diferentes cartografías se ha utilizado una serie temporal de 222 observatorios pluviométricos y 149 termométricos, obtenida en el Instituto 
Nacional de Meteorología. Para homogeneizar los promedios se siguió el planteamiento de Ninyerola et al., (2000), utilizando para la interpolación las series pluviométricas de más de 20 años de registros y las termométricas de más de 15 años. Por esta razón, la base de datos se redujo a 99 estaciones pluviométricas y 61 termométricas, de las que se calcularon los promedios totales anuales.

Se realizó un muestreo aleatorio, seleccionando el $70 \%$ de los datos para realizar las diferentes cartografías. El 30\% restante se reservó para testar los resultados.

Las diferentes interpolaciones se han realizado a un tamaño de celda de $1 \mathrm{~km}$. Se utilizó un modelo digital de elevaciones (MDE) con igual tamaño de celda, proporcionado por la Confederación Hidrográfica del Ebro, para realizar las interpolaciones en las que era precisa la variable elevación.

\subsection{Técnicas de interpolación}

En los últimos años, gracias a la aparición de herramientas informáticas y a la posibilidad de generar bases de datos referenciadas espacialmente, han surgido diferentes métodos de cartografía e interpolación automática. Estos procedimientos, basados en métodos cuantitativos y en estadística espacial, se clasifican en métodos globales, locales y métodos de interpolación óptimos o geoestadísticos (Borrough y McDonell, 2000). En la literatura científica existen innumerables ejemplos de la aplicación de cada uno de estos métodos a la interpolación de variables climáticas.

\subsubsection{Utilización de métodos de interpolación globales}

Los métodos globales más utilizados recurren a información externa para la realización de la interpolación. Son métodos inexactos en los que el valor estimado por el modelo no tiene por qué coincidir con el válor real de la variable climática en los observatorios meteorológicos donde se produce la medición (Borrough y McDonnell, 1998). Los métodos de interpolación basados en modelos empíricos de regresión simple o múltiple permiten estimar el valor de la variable climática a partir de una serie de variables independientes. Konrad II (1996) genera diferentes modelos en los que factores topográficos actúan como variables independientes explicativas respecto a eventos de precipitación de diferente intensidad. Goodale et al., (1998); Hargy (1997); Salas y Chuvieco (1993); Daly et al. (1994); Prudhomme y Reed (1998), entre otros, interpolan diferentes variables climáticas mediante la utilización de información topográfica (fundamentalmente elevación) y geográfica (dirección de los flujos de viento, distancia al mar,...). 
El primer método de carácter global aplicado a la interpolación de temperaturas y precipitaciones ha sido el ajuste de una serie de modelos empíricos lineales que se corresponden con superficies de tendencia de diferente orden (lineal, cuadrática, cúbica, orden cuatro y cinco). La forma de las primeras tres funciones es la siguiente:

Lineal:

$$
z=b_{0}+b_{1} x+b_{2} y
$$

Cuadrática:

$$
z=b_{0}+b_{1} x+b_{2} y+b_{3} x^{2}+b_{4} x y+b_{5} y^{2}
$$

Cúbica:

$$
z=b_{0}+b_{1} x+b_{2} y+b_{3} x^{2}+b_{4} x y+b_{5} y^{2}+b_{6} x^{3}+b_{7} x y^{2}+b_{8} x^{2} y+b_{9} y^{3}
$$

Donde $z$ es el valor estimado. $x$ e $y$ son las coordenadas longitud y latitud, respectivamente. $b_{\|}$son los diferentes coeficientes de las ecuaciones.

El segundo método global utilizado ha consistido en la elaboración de dos modelos empíricos de regresión múltiple para temperaturas y precipitaciones. Las relaciones que se producen entre las variables climáticas y la topografía se han estudiado de forma dilatada en la literatura científica (Bonacina, 1945; Daly et al., 1994; Basist et al., 1994; Tabony, 1985). En el caso de las precipitaciones las variables independientes utilizadas han sido la elevación, longitud y latitud. Para las temperaturas se ha introducido también la radiación solar potencial media diaria a lo largo del año obtenida según el método de Pons (1996) a partir del SIG Miramón (Pons, 1998). En la figura 2 se muestra la relación existente entre las precipitaciones anuales y las variables elevación, latitud y longitud. Existe un claro control de la precipitación por parte de la elevación del terreno, registrándose los mayores valores de la variable en los observatorios ubicados a mayor altitud. La presencia de las cadenas prepirenaicas al norte del área de estudio condiciona un mayor reparto de precipitaciones en estos sectores, debido tanto a la elevación como a la mayor apertura a las perturbaciones asociadas al frente polar. La latitud interviene positivamente en el incremento de los valores de precipitación, debido a la más frecuente llegada de precipitaciones frontales en el norte, aspecto que también se apoya en la ubicación de las mayores elevaciones en los sectores septentrionales. La existencia de algunos observatorios ubicados en bajas latitudes con altos valores de precipitación está condicionada por la presencia de sierras más elevadas y a una mayor apertura de las áreas surorientales a las perturbaciones mediterráneas. No existe una relación clara entre precipitación y longitud, si bien las áreas noroccidentales reciben una mayor cantidad de precipitaciones debido a su apertura ante los flujos dominantes de dirección N-NW. 


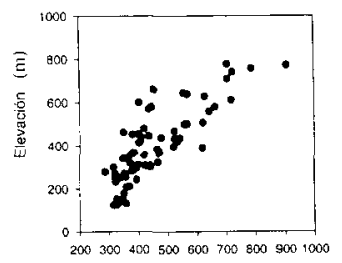

Precipitación (mm)

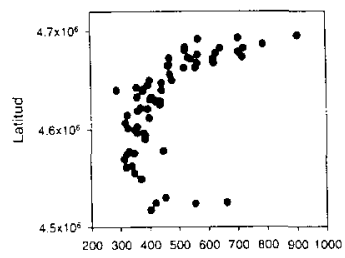

Precipitación (mm)

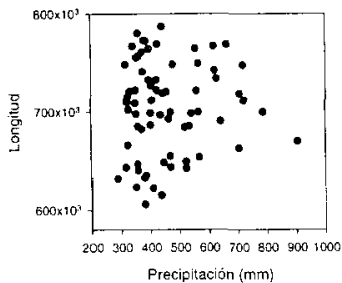

Figura 2. Relación entre las precipitaciones medias anuales y las variables elevación, latitud y longitud.

En la figura 3 se recogen las relaciones existentes entre las temperaturas medias anuales y las diferentes variables utilizadas en el modelo. Las dos únicas variables en las que se encuentra una clara relación con los valores térmicos son la elevación y la latitud, con una relación inversa en ambos casos. Las variables longitud y radiación solar incidente no influyen en la distribución espacial de las temperaturas. En el caso de la radiación solar es debido a las escasas diferencias topográficas del área de estudio, con escasos contrastes entre unas áreas y otras a la escala de este trabajo (1 km).
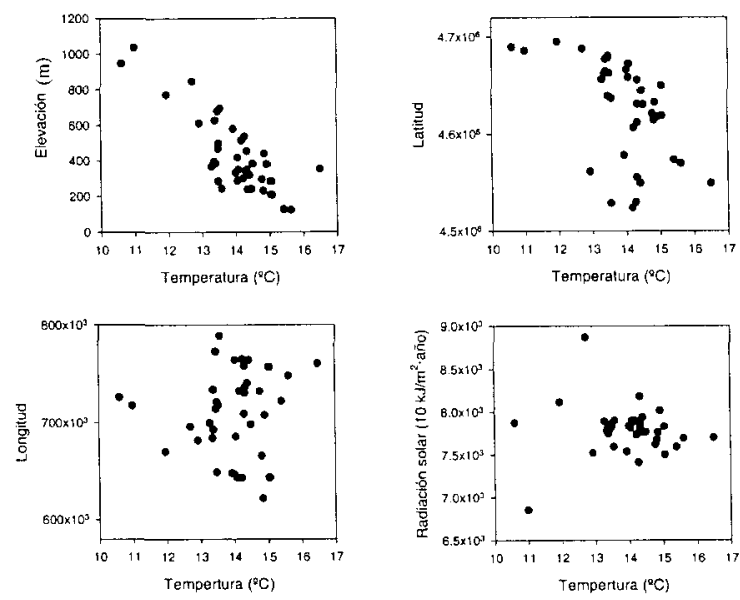

Figura 3. Relación entre las temperaturas medias anuales y las variables elevación, latitud, longitud y radiación solar potencial media diaria. 
Los resultados del análisis de regresión múltiple y los modelos obtenidos mediante un método stepwise para el caso de las precipitaciones se recogen en las tabla 1 y 2 . La inclusión de las tres variables analizadas en el modelo supone un incremento de la validez del mismo. Debido a ello, el modelo final utilizado incluye las variables elevación, longitud y latitud.

Tabla 1. Modelos obtenidos en el caso de las precipitaciones medias anuales

\begin{tabular}{|rrrrr|}
\hline MODELO & R & R CUADRADO & R CUADRADO AJUSTADO & ERROR ESTÁNDAR DE LA ESTIMACION \\
\hline A & 0.796 & 0.634 & 0.628 & 79.28523 \\
B & 0.882 & 0.778 & 0.771 & 62.20266 \\
C & 0.921 & 0.847 & 0.840 & 51.97802 \\
\hline
\end{tabular}

A Predictores: (Constante) y Elevación.

B Predictores: (Constante), Elevación y Latitud.

C Predictores: (Constante), Elevación, Latitud y Longitud.

Tabla 2. Coeficientes del modelo de regresión múltiple (precipitaciones).

\begin{tabular}{|c|c|c|c|}
\hline VARIABLES & COEFICIENTES & ERROR ESTÁNDAR & SIGNIFICACIÓN \\
\hline (Constante) & -6001.612 & 667.247 & .000 \\
\hline Elevación & .559 & .039 & .000 \\
\hline Latitud & 1.234 & .138 & .000 \\
\hline Longitud & .751 & .138 & .000 \\
\hline
\end{tabular}

Los resultados para el caso de las temperaturas se recogen en las tabla 3 y 4. Solamente se han incluido en el modelo las variables elevación y latitud. La longitud y la radiación solar potencial media diaria no son significativas.

Tabla 3. Modelos obtenidos en el caso de las temperaturas medias anuales

\begin{tabular}{|rrrrr|}
\hline MODELO & $\mathrm{R}$ & R CUADRADO & R CUADRADO AJUSTADO & ERROR ESTÁNDAR DE LA ESTIMACIÓN \\
\hline A & 0.806 & 0.649 & 0.641 & 0.6555 \\
B & 0.861 & 0.741 & 0.728 & 0.5701 \\
\hline
\end{tabular}

A Predictores: (Constante) y Elevación.

B Predictores: (Constante), Elevación y Latitud.

Las variables Longitud y Radiación solar incidente no son significativas.

Tabla 4. Coeficientes del modelo de regresión múltiple (temperaturas).

\begin{tabular}{|c|c|c|c|}
\hline VARIABLES & COEFICIENTES & ERROR ESTÁNDAR & SIGNIFICACIÓN \\
\hline (Constante) & 47.389 & 8.492 & .000 \\
\hline Elevación & $-3.724 \mathrm{E}-03$ & .000 & .000 \\
\hline Latitud & $-6.880 \mathrm{E}-03$ & .002 & .001 \\
\hline
\end{tabular}




\subsubsection{Utilización de métodos de interpolación locales}

Los interpoladores locales no utilizan información auxiliar, únicamente se sirven de los datos climáticos de los observatorios. Este tipo de interpolación requiere la selección de un área o un número de puntos mínimo para la predicción. Interpoladores locales como los polígonos de Thiessen (Thiessen, 1911), el inverso de la distancia al cuadrado o las splines también han sido utilizados en la interpolación de variables climáticas (Blenow y Persson, 1998; Hutchinson, 1995). Se han utilizado estos tres métodos locales, que constituyen interpoladores exactos, donde el valor estimado en los puntos de observación coincide con el valor real.

En el caso de los polígonos de Thiessen, los puntos en los que se quiere estimar el valor de la variable climática toman el valor del punto más cercano en el que existe observación. De esta manera se obtiene una red de polígonos condicionada por la distribución espacial de los puntos de muestreo, y donde se producen bruscas discontinuidades en el valor de la variable al pasar de un polígono a otro.

La técnica de interpolación mediante el inverso de la distancia combina las ideas de proximidad planteadas por el método de polígonos de Thiessen con los cambios graduales que se producen a partir de una superficie de tendencia (Borrough y McDonnell, 1998). Los valores estimados se obtienen con los puntos de muestreo existentes. Los puntos más cercanos tendrán un peso superior, ya que existe una ponderación en función de la distancia.

$$
z\left(x_{0}\right)=\frac{\sum_{i=1}^{n} z\left(x_{i}\right) \cdot d_{i j}^{-r}}{\sum_{i=1}^{n} d_{i j}^{-r}}
$$

$z\left(x_{i}\right)$ es el valor estimado para el punto donde se desea interpolar

$z\left(x_{i}\right)$ es igual al valor del punto donde existe observación

$d_{i j}$ es la distancia entre el punto $z\left(x_{0}\right)$ y el punto $z\left(x_{i}\right)$

$r$ es el exponente de la distancia que condiciona el resultado final de la cartografía.

Se han realizado cartografias con exponentes $r=1, r=2$ y $r=4$.

Finalmente se utilizó una interpolación mediante splines. Este método busca la función regular continua y derivable que se adapta mejor a los puntos de muestreo sin perder sus propiedades de continuidad. Esta función es una superposición de una familia de funciones parametrizables. Cada término tiene un peso importante en una región determinada y un peso nulo fuera de esta región. El valor estimado $z(x, y)$ 
estará determinado por dos términos, de tal manera que $T(x, y)$ es una función polínomica equiparable a las utilizadas en una interpolación mediante superficie de tendencia y que supone el término regularizador.

$$
z(x, y)=T(x, y)+\sum_{i=1}^{n} \lambda_{j} \cdot \psi_{j}\left(r_{i}\right)
$$

El segundo término engloba una serie de funciones radiales, donde $\lambda$, representa el conjunto de parámetros que se deben estimar y $\psi_{j}\left(r_{j}\right)$ son un conjunto de funciones conocidas. Mitasova et al., (1995) explican la forma de obtener los parámetros $\lambda$,

$$
\psi\left(r_{i}\right)=-\left[\ln \left(\frac{\varphi \cdot r_{i}}{2}\right)+E_{i} \cdot\left(\frac{\varphi \cdot r_{i}}{2}\right)+C_{E}\right]
$$

$\varphi$ es el coeficiente de tensión utilizado en la interpolación.

$C_{i}$ es la constante de Euler $(0.577215 \ldots)$

$E_{i}$ es la función exponencial

$$
r_{i}=\sqrt{\left(x-x_{i}\right)^{2}+\left(y-y_{i}\right)^{2}}
$$

Mediante el módulo INTERPNT del SIG Miramon se han realizado diferentes interpolaciones mediante splines, modificando los parámetros de tensión y regularización. Se han realizado interpolación con los parámetros $\varphi=400, \varphi=5000, T(x, y)=0$ y $T(x, y)=400$.

\subsubsection{Métodos geoestadísticos}

En los últimos años, los métodos más utilizados en climatología son los basados en técnicas geoestadísticas (Atkinson, 1997; Goovaerts, 1997; Cressie, 1990). Las estimaciones realizadas mediante los diferentes métodos de kriging se basan en una suma ponderada de las $n$ observaciones más cercanas al punto de estimación. El peso de cada uno de los puntos será escogido para que la estimación no se encuentre sesgada y tenga la menor varianza posible en relación a otras posibles combinaciones lineales entre el conjunto de observaciones disponibles. Numerosos trabajos describen minuciosamente las diferentes técnicas geoestadísticas (Borrough y McDonnell, 1998; Goovaerts, 1997; Isaaks y Strivastava, 1989). Existen diferentes técnicas geoestadísticas que incluyen una o más variables en la interpolación (simple kriging, ordinary kriging, block kriging, cokriging,...) con una complejidad creciente cuanto mayor es el número de variables introducidas. En la bibliografía encontramos múltiples ejemplos de la interpolación de variables climáticas a partir de métodos geoestadísticos (Karnieli, 1990; Phillips, et al., 1992; Ishida y Kawashima, 1993; Bacchi y Kottegoda, 1995; Bigg, 1991; Goovaerts, 2000). Diferentes resultados han sido obtenidos al aplicar las distintas técnicas geoestadísticas. Para la realización de las carto- 
grafias de precipitaciones y temperaturas de este trabajo mediante técnicas geoestadísticas se ha utilizado el programa GSTAT (Pebesma and Wesseling, 1998), tanto para los ajustes y la obtención de los semivariogramas como para la interpolación final.

\subsubsection{Métodos mixtos}

Recientemente se están desarrollando procedimientos que combinan diferentes tipos de métodos (Ninyerola et al., 2000; Prudhomme y Reed, 1999). Estos planteamientos presentan la ventaja de considerar las tendencias generales y relaciones físicas que se producen entre la variable climática y los factores geográficos del medio y tener en cuenta la correlación y dependencia espacial de la información climática disponible.

Los métodos globales son interpoladores inexactos, de tal manera que el valor estimado no se corresponde con el valor real de la variable en el punto de observación. Ninyerola et al. (2000) desarrollan un corrector relacionado con la magnitud de las diferencias en cada punto de muestreo:

\section{Corrector $=$ observado - predicho}

Los valores de corrección obtenidos se utilizan para refinar los resultados obtenidos mediante el modelo de regresión. Sin embargo, como estas correcciones se encuentran irregularmente distribuidas en el espacio es necesario interpolarlas. En el caso de las precipitaciones se han obtenido cartografías de corrección mediante inverso de la distancia al cuadrado y kriging, ya que existían unos patrones de correlación espacial entre los residuos y se pudo ajustar un semivariograma. En el caso de las temperaturas no existía dicha correlación espacial, de tal manera que se sustituyó el kriging por una interpolación mediante splines.

Mediante la suma de las cartografías de corrección y las cartografías predichas mediante el modelo de regresión (Ecuación 9) se obtienen mapas donde los valores predichos son modificados por las cartografías de corrección. De tal forma que en los lugares de observación obtendremos valores reales de la variable climática.

Observado= predicho+ corrector

\subsection{Test y validación de los resultados}

Con los datos de precipitaciones y temperaturas medias anuales de las estaciones reservadas para el test (30\% de los datos iniciales) se estableció una comparación de las estimaciones obtenidas con cada uno de los métodos de interpolación y los valo- 
res reales de los observatorios. La calidad final de cada una de las cartografías se estimó mediante el error medio cuadrático (RMSE). Cuanto más próximo sea su valor a 0 mejor será la cartografía resultante.

$$
R M S E=\sqrt{\frac{1}{n} \sum_{i=1}^{n}\left(z_{i}-z_{i}\right)^{2}}
$$

$z_{\text {e }}$ es el valor observado y el circunflejo $\wedge$ indica el valor estimado por el método correspondiente.

\section{Resultados}

En la figura 4 se indica el RMSE obtenido en cada una de las cartografías de precipitaciones y temperaturas a partir de los datos de los observatorios reservados para el test. En el caso de las precipitaciones (gráfico A), las diferencias observadas entre los diferentes métodos son muy marcadas. Las técnicas que reportan mejores resultados son las geoestadísticas, con un RMSE que oscila entre 22.02, en el caso del block kriging y 27.1 en el método de kriging universal con tendencia cuadrática. El rango de variación entre todos los métodos geoestadísticos es únicamente de $5 \mathrm{~mm}$, si bien, el block kriging y cokriging son los dos métodos que reportan mejores resultados. Tras los métodos geoestadísticos son las superficies de tendencia de 3 er y 4 " orden las que reportan mejores resultados, con un RMSE de 27.3 y 27.5 respectivamente. Entre los métodos locales, las splines, con diferentes parámetros, son la técnica que mejor resultado ofrece. Los polígonos de Thiessen y el inverso de la distancia suman errores mayores, aunque es el exponente $r=2$ el que funciona mejor (RMSE $=34.5$ ). Las superficies de tendencia de menor orden y el modelo de regresión son los métodos de peores cartografias resultantes. Aunque la introducción de mapas de corrección mejoran las estimaciones del modelo de regresión los resultados siguen quedando a mucha distancia de los métodos geoestadísticos. La interpolación de las correcciones mediante inverso de la distancia al cuadrado presenta mejores resultados que la interpolación con kriging.

La calidad de los diferentes métodos en la interpolación de las temperaturas es diferente al encontrado en la interpolación de las precipitaciones (Gráfico B). Comparativamente, el RMSE que reportan las cartografias de temperaturas es superior al de las cartografias de precipitación. La disponibilidad de un menor número de observatorios termométricos provoca mayores errores en la interpolación a partir de técnicas geoestadísticas; muy llamativos en el caso del cokriging $\left(0.8^{\circ} \mathrm{C}\right)$. En este caso los métodos locales como el inverso de la distancia al cuadrado o las splines con tensión dan mejores resultados que los diferentes tipos de kriging. De entre todos ellos, 

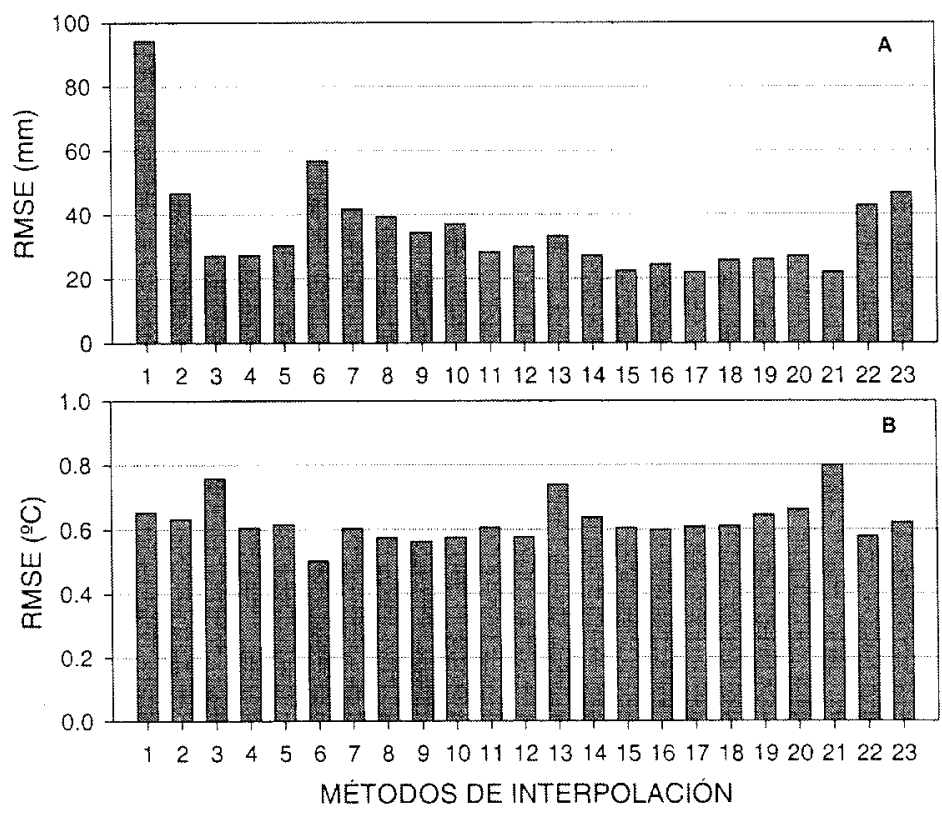

Figura 4- RMSE en $\mathrm{mm}$ y ${ }^{\circ} \mathrm{C}$ de las diferentes cartografías de precipitaciones y temperaturas medias anuales. El estadístico se obtiene mediante la comparación entre los valores reales de las estaciones reservadas para el test y los valores estimados mediante los diferentes métodos de interpolación. Las diferentes categorías hacen referencia a los siguientes métodos de interpolación: 1- Superficie de tendencia (lineal), 2- Superficie de tendencia (cuadrática), 3- Superficie de tendencia (cúbica), 4- Superficie de tendencia ( $4^{\circ}$ orden), 5- Superficie de tendencia ( $5^{\circ}$ orden), 6 Modelo de regresión, 7- Polígonos de Thiessen, 8- Inverso de la distancia $(r=1), 9$ - Inverso de la distancia $(r=2)$, 10- Inverso de la distancia $(r=4), 11$ - Splines con tensión $(\varphi=400)$, 12- Splines con tensión $(\varphi=5000)$, 13- Splines regularizadas $[T(x, y)=0]$, 14- Splines regularizadas $[T(x, y)=$ 400], 15- Simple kriging, 16- Kriging ordinario, 17- Block kriging, 18-Kriging direccional, 19Kriging universal con tendencia lineal, 20- Kriging universal con tendencia cuadrática, 21Cokriging, 22- Modelo de regresión + residuales (I.D. $r=2$ ), 23- Modelo de regresión + residuales (kriging en el caso de precipitaciones y splines en el de temperaturas).

el inverso de la distancia con exponente $r=2$, al igual que en el caso de las precipitaciones, es el método en el que la cartografía tiene mayor calidad (RMSE $=0.56^{\circ} \mathrm{C}$ ). En la interpolación mediante distintos métodos globales también se encuentran diferencias significativas. El que peores resultados reporta es la superficie de tendencia cúbica (RMSE $=0.75^{\circ} \mathrm{C}$ ). El modelo de regresión es el método cuyo RMSE entre valores observados y estimados es inferior $\left(0.5^{\circ} \mathrm{C}\right)$, de entre todos los testados, además, la introducción de mapas correctores no aporta mejoras en el modelo, sino que frente a lo esperado, se produce el fenómeno contrario. Se incrementa el RMSE, tanto en la interpolación de los residuos mediante inverso de la distancia como mediante splines. 
En la figura 5 se muestran un ejemplo cartográfico de los resultados obtenidos mediante la aplicación de los diferentes métodos de interpolación en la cartografía de las precipitaciones medias anuales. Entre las cartografías existen una serie de patrones espaciales comunes, aunque también se detectan diferencias espaciales muy marcadas. En todos los casos existe un claro gradiente pluviométrico Norte-Sur, con mayores valores pluviométricos al norte, reflejando los mayores aportes que las perturbaciones asociadas al frente polar dejan en estos sectores, igualmente aparecen altos valores de precipitación en el extremo suroriental, en un área donde las perturbaciones de carácter mediteráneo tienen una importante influencia, aspecto resaltado por la existencia de elevaciones superiores a $1.000 \mathrm{~m}$. El modelo de regresión múltiple muestra una distribución de las precipitaciones muy influida por el relieve, con una serie de discontinuidades en la transición norte-sur provocadas por accidentes topográficos menores (relieves tabulares de hasta $600 \mathrm{~m}$ s.n.m, como la sierra de Alcubierre, los montes de Zuera o la "muela" de Zaragoza).

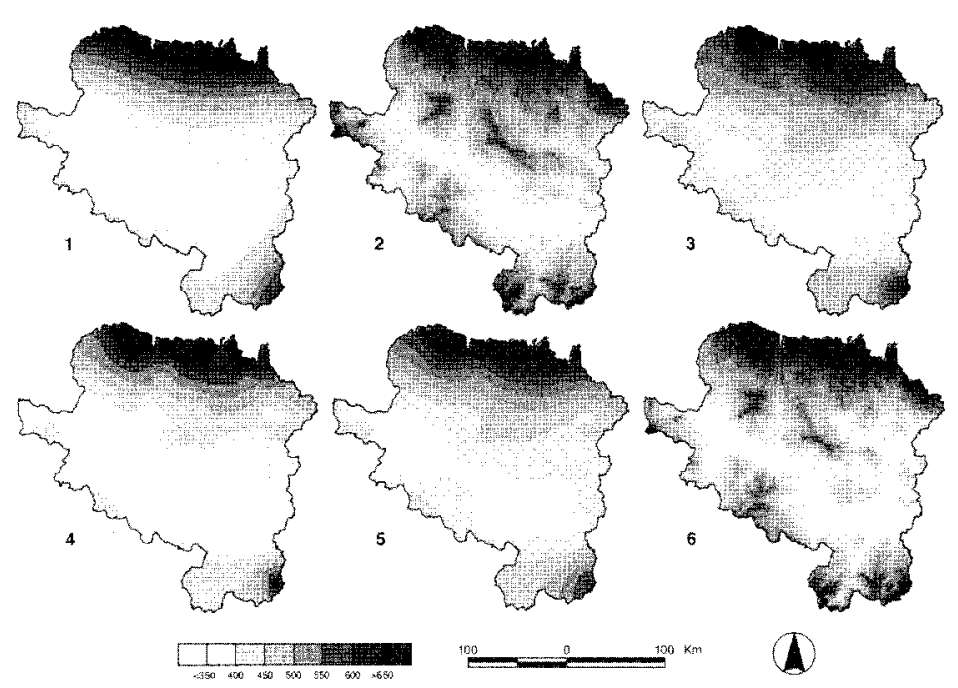

Figura 5: Ejemplo de cartografías de precipitación media anual. Datos en $\mathrm{mm}$. 1- Superficie de tendencia ( $4^{\circ}$ orden), 2 - Modelo de regresión, 3- Inverso de la distancia $(r=2)$, 4- Splines con tensión ( $\varphi=400)$, 5- Block kriging, 6- Modelo de regresión + residuales (l.D. $r=2$ ).

Los interpoladores locales muestran una mayor regularización espacial de la distribución de las precipitaciones. Los resultados de los métodos geoestadísticos difieren escasamente entre ellos y tal como muestra la cartografia obtenida mediante block. kriging existe una rápida transición norte-sur donde el gradiente pluviométrico es muy fuerte en el sector septentrional, para dar paso a una amplia área donde las precipitaciones medias anuales son muy escasas $(<350 \mathrm{~mm})$. El modelo de regresión 
corregido con los residuales muestra un aspecto similar al mapa sin corregir, aunque con algunas diferencias en el sector oriental, donde con esta corrección se obtienen menores valores de precipitación.

En la figura 6 se muestran los mismos ejemplos para el caso de las temperaturas medias anuales. La identificación de patrones generales es menos nítida que en el caso de las precipitaciones, aunque también se aprecia una gradación norte-sur, con unas temperaturas más frías en el norte del área de estudio coincidiendo con las sierras prepirenaicas y unos valores térmicos más elevados en el centro de la depresión, coincidiendo con las áreas de menor elevación. En el sureste también aparecen bajos valores térmicos, coincidiendo, de nuevo, con un incremento de la elevación en el área de los puertos de Beceite. Las diferencias espaciales que existen entre las distintas cartografías son muy importantes. Al igual que lo observado en el caso de las precipitaciones, el modelo de regresión múltiple muestra unos patrones espaciales claramente controlados por la topografía.

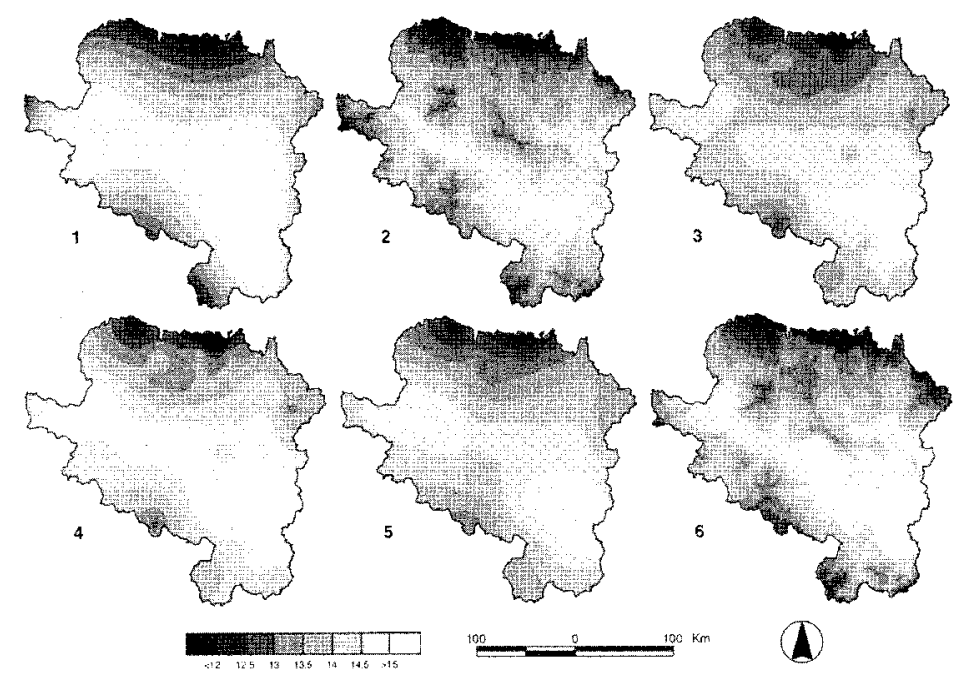

Figura 6: Ejemplo de cartografías de temperaturas medias anuales. Datos en ${ }^{\circ} \mathrm{C}$.

1- Superficie de tendencia ( $4^{\circ}$ orden), 2- Modelo de regresión, 3- Inverso de la distancia $(r=2), 4-$ Splines con tension $(\varphi=400)$, 5- Block kriging, 6- Modelo de regresión + residuales (I.D. $r=2)$.

Los métodos locales, como el inverso de la distancia al cuadrado, muestran una gran heterogeneidad espacial entre espacios cercanos como consecuencia de la distribución y los contrastes espaciales en las estaciones meteorológicas. Los diferentes métodos geoestadísiticos aplicados difieren entre si en mayor medida que en la cartografía de precipitaciones, aunque tiene lugar una mayor suavización de los patro- 
nes locales respecto a las cartografías obtenidas mediante inverso de la distancia o splines. La cartografía resultante de la aplicación de un mapa de corrección al modelo de regresión sigue manteniendo los rasgos de preponderancia del relieve, aunque se aprecian diferencias respecto al modelo original, como la mayor extensión superficial de las temperaturas, más elevadas en el centro de la depresión.

\section{Conclusiones}

Se ha comprobado que existen claras diferencias entre los métodos de interpolación más adecuados para la obtención de cartografías continuas en el caso de las precipitaciones y temperaturas medias anuales. Los factores que condicionan el reparto espacial de estas variables y la complejidad espacial a escala local son diferentes, por lo que los métodos que aportan los mejores resultados son distintos.

En el caso de las precipitaciones, debido a las escasas diferencias que existen en la aplicación de los diferentes métodos geoestadísticos se puede afirmar que en regiones con características climáticas de la complejidad que caracteriza al área de estudio podamos seleccionar aquellos métodos más sencillos que demandan menor información externa, ya que es el block kriging el que mejores resultados reporta. La distribución espacial de las precipitaciones supone que, a pesar de que exista una elevada correlación con la elevación, esta variable tenga unos efectos muy diferentes según la localización de los relieves montañosos circundantes. Tal como señalan Brundsdon et al., (2001) la relación entre precipitación y elevación varía espacialmente, siendo el efecto orográfico diferente en función del tipo de perturbación. En el norte del área de estudio las cadenas prepirenaicas reciben una mayor cantidad de precipitaciones durante las estaciones en las que predominan las perturbaciones asociadas al frente polar. Sin embargo, en el sureste, la mayor elevación condiciona la existencia de precipitaciones más altas asociadas a las borrascas de carácter mediterráneo que, de forma acumulada, aportan menores precipitaciones a lo largo del año. En las áreas más llanas también encontramos los mismos factores explicativos, pero con una mayor incertidumbre en el tipo de influencia dominante. Algunos años predominan los patrones de carácter mediterráneo, mientras que otros años son dominantes los flujos de dirección NW asociados al frente polar (Creus, 1983). Los espacios noroccidentales reciben con mayor frecuencia la influencia oceánica, que se agota rápiclamente al penetrar en el interior del valle, por lo que las diferencias en cortas distancias pueden ser muy importantes. Konrad II (1996) pone de manifiesto la complejidad de los patrones que rigen la distribución espacial de las precipitaciones a escala continental, con variaciones en el régimen, distribución y localización espacial de las pre cipitaciones según las estaciones del año y los flujos dominantes. Toda la dinámica atmosférica y la distribución espacial de las unidades de relieve supone una dificul- 
tad en la aplicación de métodos de carácter global, ya que los modelos empíricos no tienen en cuenta la diversidad local que tiene lugar como consecuencia de los complejos patrones atmosféricos. A esta complejidad se une, de forma importante, la enorme frecuencia de bajas de carácter térmico durante el periodo estival, que producen frecuentes fenómenos tormentosos en el sector central del valle, muy localizados en el espacio y con una gran importancia de los factores geográficos a escala local. Este fenómeno también dificulta la obtención de modelos empíricos más precisos que permitan una mejor cartografía del reparto espacial de las precipitaciones. Ni siquiera la corrección mediante residuales permite mejorar sustancialmente estos modelos, a pesar de que al igual que Ninyerola et al., (2000) y Prudhomme y Reed (1999) se obtengan mejores resultados mediante la introducción de mapas de corrección. Así pues, son los métodos que únicamente tienen en cuenta la distribución espacial de la variable los que reportan mejores resultados. Los métodos geoestadísticos analizan la autocorrelación espacial entre los diferentes puntos de muestreo, lo que explica que se obtengan mejores resultados que con los métodos locales.

En la interpolación de las temperaturas medias anuales se ha demostrado que el reparto de las mismas está muy condicionado por factores topográficos, fundamentalmente la elevación, y en baja medida por factores de carácter atmosférico. Por esta razón, los modelos empíricos basados en información externa presentan mejores resultados que las estimaciones realizadas a partir de métodos locales. Incluso la introducción de estos métodos en la corrección posterior del modelo no aporta mejoras sino que se reduce la calidad de las estimaciones.

Así pues, la calidad de los diferentes métodos varía en función de la variable a cartografiar. Los métodos globales (modelos de regresión) son mejores en la estimación de los valores de temperaturas medias anuales, ya que se trata de una variable con un elevado control ambiental por parte de variables como la elevación o la latitud. Las precipitaciones, en cambio, presentan comportamientos y valores diferentes en espacios de similares características topográficas o geográficas, ya que la localización de los centros de acción y de los flujos que dominan en los distintos espacios son, en muchas ocasiones, diferentes. Por esta razón, la cartografía de las precipitaciones a través de modelos de regresión múltiple aporta peores resultados que los que se obtienen mediante la cartografía con métodos geoestadísticos, ya que aunque se trata de un espacio reducido y muy liano, las diferencias geográficas dentro del mismo son significativas y más aún la diversidad y complejidad climática. El análisis de la varianza y correlación espacial que permiten los métodos geoestadísticos supone una eficaz herramienta en la estimación de las variables que tienen patrones tan complejos como son las precipitaciones, donde las diferentes variables ambientales tienen efectos diferentes según los espacios.

Se puede afirmar que dado el interés que supone una adecuada cartografia de las variables climáticas a escala local es necesario testar previamente diferentes métodos 
de interpolación para seleccionar los más adecuados en el caso de la variable climática de interés. La versatilidad que tienen los Sistemas de Información Geográfica y la actual implementación de la mayor parte de los métodos de interpolación en Sistemas de este tipo facilita el proceso de obtención de cartografías climáticas, y nos permite llevar a cabo estimaciones fiables y fácilmente integrables en modelos agrícolas, en la gestión territorial o en el perfeccionamiento de los modelos climáticos a escalas locales.

\section{Agradecimientos}

Este trabajo ha contado con el apoyo del proyecto de investigación CLI99-0981, financiado por la Comisión Interministerial de Ciencia y Tecnología (CICYT), Plan nacional de I+D. Agradecer al Instituto Nacional de Meteorología (INM) la disponibilidad de los datos utilizados para este trabajo y a la Confederación Hidrográfica del Ebro (CHE) la cesión del Modelo Digital de Elevaciones utilizado en el trabajo. Agradecer a los revisores anónimos las valiosas aportaciones que han realizado al manuscrito original.

\section{Bibliografía}

Ascaso, A y Casals, M., (1981): Periodos secos y sequías en la depresión central del Ebro. Geographicalia, 11-12, p. 55-71.

Atkinson, P.M., (1997): Geographical information science. Progress in Pbysical Geograpby 21, p. 573-582.

Bacchi, B. y Kottegoda, N.T., (1995): Identification and calibration of spatial correlation patterns of rainfall. Joumal of Hydro$\log y$. 165, p. 311-348.

Basist, A., Bell, G.D. y Meentemeyer, V., (1994): Statistical Relationships between topography and precipitation patterns. Journal of Climate. 7, p. 1305-1315.
Bigg, G.R., (1991): Kriging and intraregional rainfall variability in England. International Journal of Climatology. 11, p. 663-675.

Blennow, K. y Persson, P., (1998): Modelling local-scale frost variations using mobile temperature measurements with a GIS. Agricultural and Forest Meteorology 89, p. 59-71.

Bonacina, L.C.W., (1945): Orographic rainfall and its place in the hydrology of the globe. Quarterly fournal of the Royal Meteorological Society, 71, p. 41-55.

Borrough, P.A. y McDonnell, R.A., (1998): Principles of Geographical Information Systems. Oxford. Oxford University Press. 
Brundsdon, C., McClatchey, J. y Unwin, D.J., (2001): Spatial variations in the average rainfall-altitude relationship in Great Britain: an approach using geographically weighted regression. International Journal of Climatology 21, p. 455-466.

Cressie, N., (1990): The origins of kriging. Mathematical Geology 22, p. 239-253.

Creus, J., (1983): El clima del alto Aragón occidental. Instituto de estudios Pirenaicos. Serie Monografias, 109. Jaca.

Creus, J., (1996): variaciones en la disponibilidad hídrica mensual en el valle del Ebro. En Clima y agua: la gestión de un recurso climático (M.V. Mrzol, P. Dorta and P. Valladares Eds.) Universidad de la Laguna, p. 79-86.

Creus, J. y Ferraz, J, (1995): Irregularidad pliviométrica y continentalidad térmica en el valle medio del Ebro. Lucas Mallada, 7 , p. 147-164.

Cuadrat, J.M., (1989): Las oscilaciones climáticas recientes en Zaragoza (1865-1984). Geograpbicalia, 26, p. 53-60.

Cuadrat, J.M., (1991): La sequía en el valle del Ebro. Aspectos climáticos y consecuencias económicas. Revista de la Real Academia de Ciencias, 85, p. 537-545.

Cuadrat, J.M., (1999): El clima de Aragón. Caja de Ahorros de la Inmaculada. Zaragoza. $109 \mathrm{pp}$.

Daly, C., Neilson, R.P. y Phillips, D.L., (1994): A statistical-topographical model for mapping climatological precipitation over mountainous terrain. Joumal of Climate and Applied Meteorology 33, p. 140-158.

Del Valle, J., (1996): El clima del Prepirineo central y occidental aragonés $y$ sus somontanos. Consejo de protección de la naturaleza de Aragón. Zaragoza. 327 pp.

Goodale, C.L., Aber, J.D. y Ollinger, S.V., (1998): Mapping monthly precipitation, temperature and solar radiation from Ireland with polynomial regression and a digital elevation model. Climate Research 10 , p. $35-49$.

Goovaerts, P., (1997): Geoestatistics for natural resources evaluation. Oxford University Press, New York.

Goovaerts, P., (1999): Using elevation to aid the geostatistical mapping of rainfall erosivity. Catena 34, p. 227-242.

Goovaerts, P., (2000): Geostatistical approaches for incorporating elevation into the spatial interpolation of rainfall. Journal of bydrology. 228, p. 113-129.

Hargy, V.T., (1997): Objectively mapping accumulated temperature for Ireland. International Joumal of Climatology. 17, p. 909-927.

Hutchinson, M.F., (1995): Interpolating mean rainfall using thin plate smoothing splines. International Journal of Geographical Information Systems 9, p. 385-403.

Isaaks, E.H. y Strivastava, R.M., (1989): An introduction to Applied Geostatistics. Oxford University Press. Oxford.

Ishida, T. y Kawashima, S., (1993): Use cokriging to estimate surface air temperature from elevation. Theoretical and Applied Climatology. 47, p. 147-157.

Karnieli, A., (1990): Application of kriging technique to areal precipitation mapping in Arizona. Geojoumal 22 (4), p. 391-398.

Konrad II, C.E., (1996): Relationships between precipitation event types and topography 
in the southern blue ridge mountains of the southeastern USA. International Joumal of Climatology. 16, p. 49-62.

Mitasova, H., Mitas, L., Brown, W.M., Gerdes, D.P., Kosinovsky, I. y Baker, T., (1995): Modelling spatially and temporally distributed phenomena: new methods and tools for GRASS GIS. International Journal of Geograpbical Information Systems. 9 (4), p. 433-446.

Ninyerola, M., Pons, X. y Roure, J.M., (2000): A methodological approach of climatological modelling of air temperature and precipitation through GIS techniques. International Journal of Climatology. 20, p. 1823-1841.

Pebesma, E.J. y Wesseling, C.G., (1998): Gstat, a program for geostatistical modelling, prediction and simulation. Computers and Geosciences, 24 (1), p. 17-31.

Pedrocchi, C., (1998): Ecología de los Monegros. Instituto de Estudios Altoaragoneses. $430 \mathrm{pp}$.

Pellicer, F., y Echeverría, M.T., (1990): Formas de relieve del centro de la Deprestón del Ebro. Institución Fernando el Católico, 216 pp.

Pérez, J. y Creus, J., (1994): Tendencia secular de la precipitación en Zaragoza (18651984). En Cambios y variaciones climáticas en España. (M.F. Pita and M. Aguilar Eds.). Universidad de Sevilla, p. 169-182.

Phillips, D.L., Dolph, J. y Marks, D., (1992): A comparison of geostatistical procedures for spatial analysis of precipitation in montainous terrain. Agricultural and Forest Meteorology. 58, p. 119-141.

Pons, X., (1996): Estimación de la radiación solar a partir de modelos digitales de ele- vaciones. Propuesta metodológica. En VII Coloquio de Geografia Cuantitativa, Sistemas de Información Geográfica $y$ Teledetección (J. Juaristi and I. Moro Eds.). Vitoria, p. 87-97.

Pons, X., (1998): Manual of Miramon. Geograpbic Information System and Remote Sensing Software.

(http://www.creaf.uab.es/miramon). Centre de Recerca Ecològica i Aplicacions Forestals (CREAF): Bellaterra; 150.

Prudhomme, C. y Reed, D.W., (1998): Relationships between extreme daily precipitation and topography in a mountainous region: a case study in Scotland. International Journal of Climatology. 18, p. 1439-1453.

Prudhomme, C. y Reed, D.W., (1999): Mapping extreme rainfall in a mountainous region using geostatistical techniques: a case study in Scotland. International Joumal of Climatology. 19, p. 1337-1356.

Salas, J. y Chuvieco, E., (1993): Modelización de variables microclimáticas a partir de un sistema de información geográfica. $2^{\circ}$ Congreso de la Asociación Española de Sistemas de Información Geográfica. Madrid. 2-4 de junio de1993, p. 423-446.

Suárez, F., Sainz, H., Santos, T. y Bernáldez, F.G., (1992): Las estepas ibéricas. Ministerio de Obras públicas y Transportes. Madrid. 160 pp.

Tabony, R.C., (1985): Relations between minimum temperature and topography in Great Britain. Journal of Climatology, 5 , p. 503-520.

Thiessen, A.H., (1911): Precipitation averages for large areas. Monthly Weather Review. 39 (7), p. 1082-1084. 